\title{
Risk Factors of Arterial Damage Assessed by ABI and baPWV among Hemodialysis Patients in Macau
}

\author{
Mingxin Li, ${ }^{1,2}$ Jing Xin, ${ }^{1,2}$ Ding-Wei Kuang, ${ }^{1,2}$ Un-I Kuok, ${ }^{1}$ Chiu-Leong Li, \\ Kin Cheung, ${ }^{1}$ and Weng-In Lio ${ }^{1}$ \\ ${ }^{1}$ Division of Nephrology, Centro Hospitalar Conde de São Januário, Macau \\ ${ }^{2}$ Division of Nephrology, Huashan Hospital, Fudan University, Shanghai 200040, China \\ Correspondence should be addressed to Mingxin Li; limingxin1969@126.com and Un-I Kuok; kuokuni@ssm.gov.mo
}

Received 26 April 2014; Revised 1 July 2014; Accepted 25 August 2014; Published 9 September 2014

Academic Editor: Francois Madore

Copyright (C) 2014 Mingxin Li et al. This is an open access article distributed under the Creative Commons Attribution License, which permits unrestricted use, distribution, and reproduction in any medium, provided the original work is properly cited.

Cardiovascular disease (CVD) is the leading cause of mortality and morbidity among patients with ESRD on chronic dialysis. Arterial damage is one of the characteristics of CVD. But the association between arterial damage and conventional risk factors for CVD has not yet been fully highlighted in chronic hemodialysis patients. Here we validate the clinical value of assessment of arterial damage by ABI and PWV in chronic hemodialysis patients in Macau.

\section{Background}

Cardiovascular disease (CVD) is the leading cause of mortality and morbidity among patients with ESRD on chronic dialysis [1]. According to the US Renal Data System and Hong Kong Renal Registry reports, CVD accounts for approximately $40 \%$ of mortality in dialysis patients $[2,3]$.

Arterial damage, including stenosis and stiffness of arteries, is one of the characteristics of CVD. Several noninvasive measurements are introduced to identify the abnormal structure and function of arteries [4]. Ankle-brachial index (ABI) serves as a reliable sign for diagnosing peripheral artery disease. A reduced ABI relates to further development of angina, myocardial infarction, stroke, and need for coronary bypass surgery. And it is a powerful independent predictor of all-cause and cardiovascular mortality in hemodialysis (HD) patients $[5,6]$. Pulse wave velocity (PWV), which provides a comprehensive assessment of arterial stiffness, is also a powerful and independent predictor of all-cause and cardiovascular mortality in ESRD patients $[7,8]$.

Classic cardiovascular risk factors, such as blood pressure, diabetes mellitus, and hyperlipidemia, have been implicated in accelerated arterial damage. However, findings of many studies in general population were inconsistent with respect to risk factors other than age and blood pressure
[9]. And the association between arterial damage and those risk factors has not yet been fully highlighted in chronic hemodialysis patients. In consideration of the genetic contribution to arterial damage [10], it is meaningful to validate the clinical value of assessment of arterial damage by $\mathrm{ABI}$ and PWV in chronic hemodialysis patients in Macau.

\section{Methods}

2.1. Subjects. We retrospectively studied all chronic HD patients who were aboriginal inhabitants of Macau (all of the enrolled patients were born in Macau and in most of time they lived in Macau) in this cross-sectional study from March 2012 to June 2012. Patients who met the following criteria were enrolled.

Entry Criteria. These include age of 18 or older, 1 year or more of duration of dialysis, and dry weight being kept stable for at least 3 months before enrollment.

Exclusion Criteria. These include presence of clinically overt congestive heart failure (NYHA class III-IV); persistent hypotension despite pharmacological therapy (defined as systolic blood pressure $(\mathrm{SBP})<90 \mathrm{mmHg}$ or diastolic blood 
pressure (DBP) $<60 \mathrm{mmHg}$ ); atrial fibrillation; acute infectious disease; liver cirrhosis with ascites; tumor; malnutrition; bilateral amputation of lower limbs; and bilateral arteriovenous fistula over arms (patients who had bilateral arteriovenous fistula over arms were not enrolled in this study).

Informed consents were obtained before enrollment. And this study was approved by the Ethical Committee of Centro Hospitalar Conde de São Januário.

\subsection{Demographic and Clinical Data Collection}

Data Included. The data included were age, gender, body weight, height, blood pressure, illness history, and etiology of ESRD.

2.3. Treatment of Uremia. Four-hour HD thrice weekly and the target of KT/V should be higher than 1.4. EPO and iron were prescribed to keep the pre-HD level of $\mathrm{Hb}$ within the range of 10-12 g/dL. Calcium, Vitamin D and its analogy, and phosphate binder were given individually or collectively to meet the target range of calcium, phosphate, and $\mathrm{PTH}$ which were $2.1-2.5 \mathrm{mM}, 1.13-1.78 \mathrm{mM}$, and 2-9 times of the upper limit of normal, respectively.

2.4. Biochemical Tests. Fasting blood samples were collected in the morning. Measurements were performed using routine laboratory methods for such serum parameters as creatinine, calcium, phosphate, albumin, blood glucose, HbA1C, total cholesterol, triglyceride, and low- and high-density lipoprotein. Serum intact parathyroid hormone (iPTH) was measured by Nichols immunoradiometric assay.

2.5. ABI Measurement. The ABI was measured using VP-1000 vascular profiler (Nippon Colin Ltd., Komaki, Japan) in HD patients before hemodialysis and at least 15-minute supine rest (the time of measurement was not specialized). ABI was automatically calculated as the ratio of ankle SBP to brachial SBP for each side and the lower value was used for analysis [11]. All the ABI measurements were performed by one experienced operator and the intraobserver coefficient of variation was about $2.68-4.45 \%$.

2.6. PWV Measurement. The brachial-ankle PWV (baPWV) was assessed using VP-1000 vascular profiler (Nippon Colin Ltd., Komaki, Japan) in HD patients before hemodialysis and at least 15-minute supine rest (the time of measurement was not specialized; PWV may change significantly after ultrafiltration measurement is taken before hemodialysis), which allowed online pulse wave recording and automatic calculation of PWV. Briefly, baPWV was calculated from $(D 1-D 2) / T$. D1 is the distance between the heart and ankle, $D 2$ is the distance between the heart and brachium, and $T$ is the transit between the right brachial arterial wave and right tibial arterial wave. The distances between the sampling points are automatically calculated from the patient's height and are divided by the time interval for the waveform from each measuring point [12]. The baPWV was performed in HD patients before hemodialysis and at least 15-minute supine rest. Two measurements were performed in each arm, and the average value was used for the analysis. All the PWV measurements were performed by one experienced operator and the intraobserver coefficient of variation was about 1.58 $3.36 \%$.

2.7. Statistical Analysis. Continuous variables with normal distribution were expressed as means \pm standard deviation. Univariate analysis was done to explore relationships between 2 variables by Pearson correlation test for data with bivariate normal distribution and Spearman rank correlation test for nonparametric data. Stepwise multiple linear regression analysis was used to assess the independent determinants of $\mathrm{ABI}$ and baPWV. A two-tailed $P<0.05$ was considered as statistically significant. All statistical analyses were performed with the statistical software Stata 7.0 (Computer Resource Center, USA).

\section{Results}

3.1. Patient Characteristics. A total of 312 maintenance HD patients (176 males/136 females) were enrolled. Etiology of ESRD is diabetic nephropathy $(n=111)$, lupus nephritis $(n=$ $4)$, primary nephritis $(n=72)$, hypertensive nephrosclerosis $(n=53)$, chronic interstitial nephritis $(n=3)$, and unknown etiology $(n=41)$. Vascular access type is arteriovenous fistula $(n=254)$, permanent catheter $(n=57)$, and arteriovenous graft $(n=1)$. Table 1 shows the demographic and clinical characteristics of the enrolled HD patients. On average of all, $\mathrm{ABI}$ was $1.03 \pm 0.20$ and baPWV was $2037.14 \pm 653.14 \mathrm{~cm} / \mathrm{s}$.

3.2. Univariate Analysis. Table 2 shows the effects of exposure factors on $\mathrm{ABI}$ and baPWV. Risk factors for ABI include age $(P=0.000)$, male $(P=0.000)$, high pulse pressure $(P=$ $0.037)$, and hyperglycemia $(P=0.007)$. Risk factors for PWV include age $(P=0.000)$, high pulse pressure $(P=0.000)$, hyperglycemia $(P=0.030)$, and hyperphosphatemia $(P=$ $0.030)$. The impact of heart rate on arterial stiffness was not probed in consideration of the variability in heart rate.

3.3. Multiple Linear Regression Analysis. In a stepwise multiple linear regression analysis, we employed ABI and baPWV value as a dependent variable, while using age, gender, HD duration, BMI, SBP (systolic blood pressure), DBP (diastolic blood pressure), MAP (mean arterial pressure), PP (pulse pressure), FBG (fasting blood glucose), HbAlc (hemoglobin Alc), cholesterol, triglycerides, LDL-C (low density lipoprotein cholesterol), HDL-C (high density lipoprotein cholesterol), Ca (calcium), P (phosphorus), Ca $\times \mathrm{P}$ (Calciumphosphate product), PTH, KT/V (index of urea clearance), and RRF (residual renal function) as independent variables (Table 3).

Risk factors for decreased $A B I$ : every 1 unit there is an increase of age; SBP, DBP, PP, FBG, Ca $\times$ P, and ABI will be reduced by $0.005(P=0.000), 0.002(P=0.001),-0.002$ $(P=0.002), 0.004(P=0.000), 0.001(P=0.011)$, and 0.034 $(P=0.037)$, respectively. For male, ABI will be reduced by 
TABLE 1: Baseline characteristics of the study population.

\begin{tabular}{lc}
\hline Items & Total $(n=312)$ \\
\hline Age (years) & $61.47 \pm 14.42$ \\
Gender (female) & $45.39 \%(136)$ \\
Occupation & $25.96 \%(81)$ \\
Married & $76.6 \%(239)$ \\
Ever smoker & $3.52 \%$ \\
Previous CVD history & $17.3 \%(54)$ \\
Diabetes mellitus & $35.58 \%$ \\
HD duration (months) & $66.97 \pm 66.72$ \\
SBP (mmHg) & $147.88 \pm 26.52$ \\
DBP (mmHg) & $78.71 \pm 15.13$ \\
PP (mmHg) & $69.17 \pm 21.32$ \\
MAP (mmHg) & $101.76 \pm 16.92$ \\
BMI (kg/m $\left.{ }^{2}\right)$ & $22.32 \pm 4.29$ \\
RAS inhibitor & $33.97 \%$ \\
Hyperlipidemia & $20.19 \%$ \\
Serum albumin (g/L) & $43.58 \pm 4.52$ \\
Cholesterol (mmol/L) & $4.36 \pm 1.06$ \\
Triglycerides (mmol/L) & $1.79 \pm 1.21$ \\
LDL cholesterol (mmol/L) & $2.26 \pm 0.87$ \\
HDL cholesterol (mmol/L) & $1.30 \pm 0.45$ \\
Hemoglobin (g/dL) & $11.57 \pm 1.56$ \\
Hematocrit $(\%)$ & $34.41 \pm 5.26$ \\
Calcium (mmol/L) & $2.46 \pm 0.21$ \\
Phosphate (mmol/L) & $1.61 \pm 0.51$ \\
Calcium-phosphate product $\left[(\mathrm{mmol} / \mathrm{L})^{2}\right]$ & $3.92 \pm 1.22$ \\
Intact-PTH (pg/mL) & $366.98 \pm 423.97$ \\
CRP & $1.09(0.12,25.2)$ \\
Kt/V & $1.54 \pm 0.34$ \\
ABI & $1.03 \pm 0.20$ \\
PWV (cm/s) & $2037.14 \pm 653.14$ \\
\hline
\end{tabular}

0.059 , as compared to female $(P=0.002)$. Liner correlation between LDL-C and ABI was found in patients whose level of LDL-C was higher than $2.6 \mathrm{mmol} / \mathrm{L}$. Every 1 unit increase of LDL-C, ABI will be reduced by $0.088(P=0.006)$.

Risk factors for increased baPWV: every 1 unit there is an increase of age; $\mathrm{BMI}, \mathrm{MAP}, \mathrm{PP}, \mathrm{Ca} \times \mathrm{P}$, and baPWV will be increased by $12.028(P=0.000), 23.779(P=0.003), 3.664$ $(P=0.044), 5.982(P=0.017)$, and $7.183(P=0.026)$, respectively. Liner correlation between LDL-C and baPWV was found in patients whose level of LDL-C was higher than $2.6 \mathrm{mmol} / \mathrm{L}$. Every 1 unit increase of LDL-C, baPWV will be increased by $214(P=0.0022)$.

\section{Discussion}

A multitude of traditional and uremia-specific risk factors may cause abnormality of structure and function of arteries [13], which would lead to arterial stenosis and stiffness. ABI and PWV are introduced in the present study in view of their potential advantages in predicting arterial damage before the onset of clinical diseases.
Although carotid-femoral PWV (cfPWV) is considered as a well-established index of central arterial stiffness [14], there are some obvious limitations [15]. Namely, it is difficult for clinical operator to use pressure transducers on target arteries. And it is unacceptable for some subjects to expose inguinal area during the acquisition of femoral pressure waveforms. BaPWV was used in place of cfPWV in the present study in consideration of the fact that baPWV reflects both central and peripheral arterial stiffness [16].

It was indicated that arterial structure was in part influenced by genetic factors [10]. To avoid the interference of heredity and environment as far as possible, we conducted this study only in aboriginal inhabitants of Macau.

Men have a higher risk of getting heart disease than women who are still menstruating. Gender is one of the CVD risk factors that cannot be changed. Data showed that, for male, ABI will be reduced by 0.059 , as compared to female $(P=0.002)$.

Age-related structural change of arteries mainly involves the media of aorta. Consistent with the previous study that there was a linear relationship between age and stiffness of central artery [17], our study also revealed age-related peripheral arterial disease in hemodialysis patients.

A negative correlation between pulse pressure and ABI, while a positive one between pulse pressure and baPWV, was confirmed, respectively, in this study, as the result of the large epidemiological study in general population [18]. Hypertension may cause arterial damage and inversely sclerotic change of arteries may induce hypertension. So it is not difficult to understand that hypertension serves as both a risk factor and a marker of impaired vascular compliance.

Hypercholesterolemia, as one of classic cardiovascular risk factors, has been implicated in accelerated arterial stiffening. However, it was not the determinant of accelerated arterial stiffness in normotensive subjects and in treated hypertensive subjects [19]. In our study, the correlation between LDL-C and arterial damage cannot be demonstrated in univariate analysis in which subjects were divided in 2 groups according to the level of LDL-C with the cut point of $2.6 \mathrm{mmol} / \mathrm{L}$. But in multiple regression analysis, in patients with LDL-C higher than $2.6 \mathrm{mmol} / \mathrm{L}$, a linear relationship was found between LDL-C and ABI or baPWV. The possible explanation may be that the relationship between LDL-C and ABI or baPWV is not completely linear, and both high and low level of LDL-C would relate to arterial damage in hemodialysis patients. In keeping with this point, it was reported that there was increased risk of CVD in dialysis patients with the lowest level of blood lipids [20].

To compare with hemoglobin Alc (HbAlc), test of blood glucose gives a more accurate picture of diabetes control in dialysis patients. A lower level of HbAlc would be found in dialysis patients, because shorter life span of red cells reduces the time that glucose interacts with hemoglobin in the bloodstream [21]. This explains why blood glucose, instead of HbAlc, was associated with ABI and baPWV in the present study.

Our research suggests that serum calcium had no correlation with arterial damage, while calcium-phosphate product showed a risk trend of arterial damage, similar to that seen 


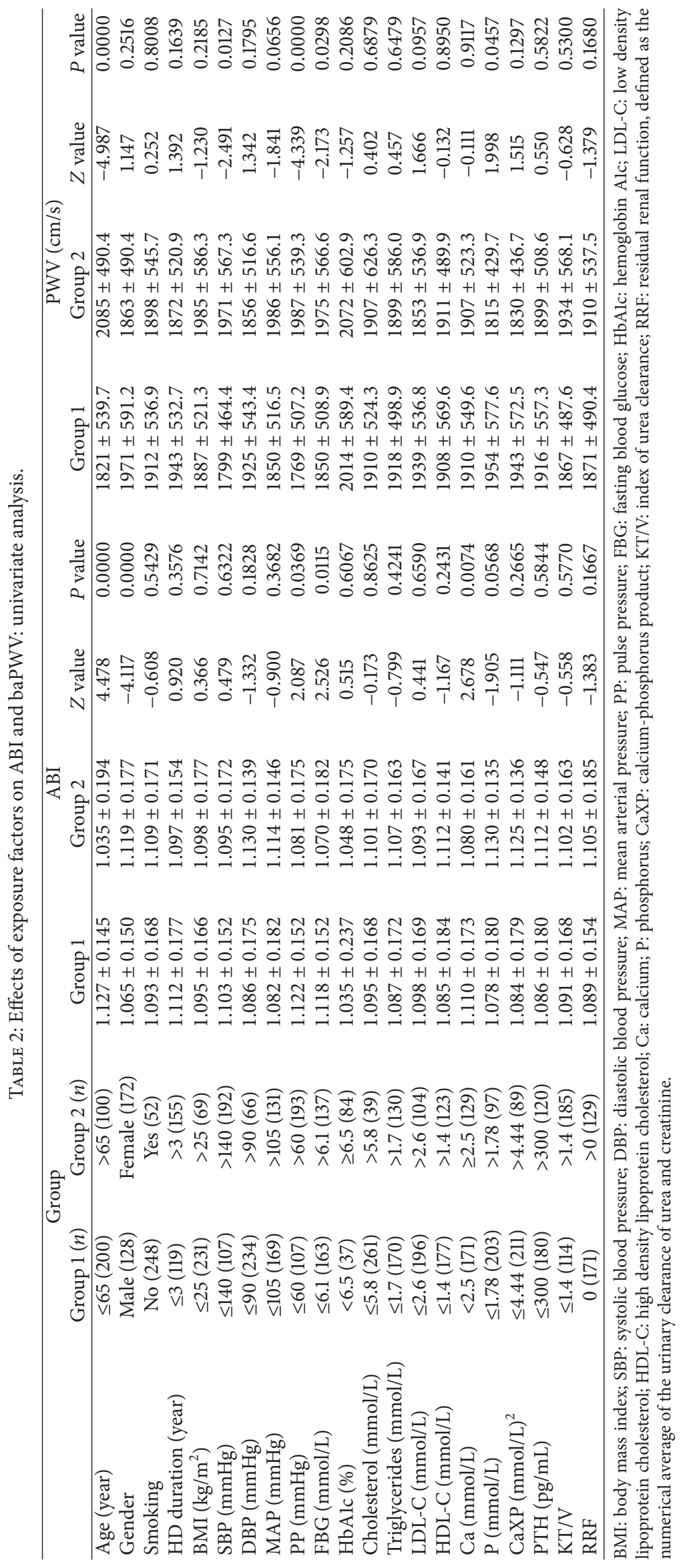


TABLE 3: Multiple linear regression analysis of the factors associated with arterial damage.

\begin{tabular}{|c|c|c|c|c|c|c|}
\hline & \multicolumn{3}{|c|}{$\mathrm{ABI}$} & \multicolumn{3}{|c|}{ baPWV } \\
\hline & Coefficient & $t$ value & $P$ value & Coefficient & $t$ value & $P$ value \\
\hline Age & -0.005 & -6.570 & 0.000 & 12.028 & 5.010 & 0.000 \\
\hline HD duration (year) & 0.001 & 0.970 & 0.332 & -1.827 & -0.580 & 0.564 \\
\hline Gender (male) & -0.059 & 2.43 & 0.002 & 19.560 & 0.270 & 0.784 \\
\hline BMI $\left(\mathrm{kg} / \mathrm{m}^{2}\right)$ & -0.001 & -0.380 & 0.706 & 23.779 & 3.040 & 0.003 \\
\hline $\mathrm{SBP}(\mathrm{mmHg})$ & -0.002 & 3.240 & 0.001 & 0.208 & 0.100 & 0.917 \\
\hline DBP (mmHg) & 0.002 & 3.050 & 0.002 & 0.527 & 0.260 & 0.797 \\
\hline MAP (mmHg) & 0.001 & 1.320 & 0.187 & 3.664 & 2.020 & 0.044 \\
\hline $\mathrm{PP}(\mathrm{mmHg})$ & -0.004 & -4.590 & 0.000 & 5.982 & 2.410 & 0.017 \\
\hline FBG $(\mathrm{mmol} / \mathrm{L})$ & -0.001 & -2.600 & 0.011 & 0.539 & 0.400 & 0.687 \\
\hline HbAlc (\%) & -0.009 & -0.930 & 0.353 & 25.780 & 0.860 & 0.394 \\
\hline Cholesterol (mmol/L) & 0.001 & 0.090 & 0.929 & -7.664 & -0.550 & 0.584 \\
\hline Triglycerides $(\mathrm{mmol} / \mathrm{L})$ & 0.005 & 0.440 & 0.661 & 47.998 & 1.550 & 0.123 \\
\hline LDL-C (mmol/L) & -0.026 & -1.890 & 0.060 & 9.675 & 0.230 & 0.816 \\
\hline $\mathrm{HDL}-\mathrm{C}(\mathrm{mmol} / \mathrm{L})$ & 0.015 & 0.510 & 0.612 & 126.769 & 1.430 & 0.155 \\
\hline $\mathrm{Ca}(\mathrm{mmol} / \mathrm{L})$ & -0.001 & -0.440 & 0.662 & 0.441 & 0.240 & 0.809 \\
\hline $\mathrm{P}(\mathrm{mmol} / \mathrm{L})$ & -0.0559 & 3.000 & 0.003 & 88.645 & 1.47 & 0.142 \\
\hline $\mathrm{CaXP}(\mathrm{mmol} / \mathrm{L})^{2}$ & -0.034 & 2.100 & 0.037 & 7.183 & 0.130 & 0.026 \\
\hline PTH (pg/mL) & 0.001 & 1.590 & 0.113 & 0.061 & 0.600 & 0.551 \\
\hline
\end{tabular}

BMI: body mass index; SBP: systolic blood pressure; DBP: diastolic blood pressure; MAP: mean arterial pressure; PP: pulse pressure; FBG: fasting blood glucose; HbAlc: hemoglobin Alc; LDL-C: low density lipoprotein cholesterol; HDL-C: high density lipoprotein cholesterol; Ca: calcium; P: phosphorus; CaXP: calcium-phosphorus product.

with serum phosphorus in the univariate analysis. And the risk associated with calcium-phosphate product was independent of PTH. These findings are consistent with the previous national study that revealed the association of calciumphosphate product with mortality in hemodialysis patients [22]. It is especially true in dialysis patients that elevated calcium-phosphate product promotes the accumulation of calcium-phosphate crystals in the media or intima of the arterial wall, which would result in arterial damage. Both high and low level of PTH may influence the metabolism of calcium and phosphate. The relationship between PTH and $\mathrm{ABI}$ or baPWV is not completely linear either.

Several limitations of this study must be taken into consideration when interpreting the data. Firstly, cross-sectional design of the study did not allow us to determine causality. Secondly, due to the technical limitation of baPWV measurement, patients with atrial fibrillation or amputated extremity were excluded. However, these patients generally are at high risk of arterial damage. Thirdly, the age on the start of dialysis was not analyzed due to relatively small sample size. Fourthly, the long term impact of different vascular access on arterial stiffness is an interesting question and needs further study.

\section{Conclusions}

In conclusion, the presented data clearly showed that age, pulse pressure, LDL-C, fasting blood glucose, and calciumphosphate product are the risk factors of arterial damage in hemodialysis patients in Macau.

\section{Conflict of Interests}

The authors declare that they have no conflict of interests.

\section{Authors' Contribution}

Mingxin Li and Jing Xin are equal contributors.

\section{Acknowledgment}

This study was supported by a Grant sponsored from Science and Technology Development Fund of Macau SAR (no. 0932011-A).

\section{References}

[1] M. S. Sidhu and K. C. Dellsperger, "Cardiovascular problems in dialysis patients: impact on survival," Advances in Peritoneal Dialysis, vol. 26, pp. 47-52, 2010.

[2] United States Renal Data System: USRDS 2010 Annual Data Report: Atlas of End-Stage Renal Disease in the United States, United States Department of Health and Human Services, Public Health Service, National Institutes of Health, National Institute of Diabetes and Digestive and Kidney Diseases, Division of Kidney, Urology, and Hematologic Diseases, Bethesda, Md, USA, 2010.

[3] Y.-W. Ho, K.-F. Chau, B.-Y. Choy et al., "Hong Kong renal registry report 2010," Hong Kong Journal of Nephrology, vol. 12, no. 2, pp. 81-98, 2010. 
[4] "The task force for the management of arterial hypertension of the European Society of hypertension (ESH) and of the European Society of Cardiology (ESC): 2007 guidelines for the management of arterial hypertension," European Heart Journal, vol. 28, pp. 1462-1536, 2007.

[5] A. Kato, T. Takita, M. Furuhashi, H. Kumagai, and A. Hishida, "A small reduction in the ankle-brachial index is associated with increased mortality in patients on chronic hemodialysis," Nephron. Clinical practice, vol. 114, no. 1, pp. c29-37, 2010.

[6] K. Ono, A. Tsuchida, H. Kawai et al., "Ankle-brachial blood pressure index predicts all-cause and cardiovascular mortality in hemodialysis patients," Journal of the American Society of Nephrology, vol. 14, no. 6, pp. 1591-1598, 2003.

[7] G. M. London, A. P. Guérin, S. J. Marchais, F. Métivier, B. Pannier, and H. Adda, "Arterial media calcification in end-stage renal disease: Impact on all-cause and cardiovascular mortality," Nephrology Dialysis Transplantation, vol. 18, no. 9, pp. 1731-1740, 2003.

[8] S.-C. Chen, J.-M. Chang, W.-C. Liu et al., "Brachial-ankle pulse wave velocity and rate of renal function decline and mortality in chronic kidney disease," Clinical Journal of the American Society of Nephrology, vol. 6, no. 4, pp. 724-732, 2011.

[9] M. Cecelja and P. Chowienczyk, "Dissociation of aortic pulse wave velocity with risk factors for cardiovascular disease other than hypertension: a systematic review," Hypertension, vol. 54, no. 6, pp. 1328-1336, 2009.

[10] F. A. Sayed-Tabatabaei, M. J. E. van Rijn, A. F. C. Schut et al., "Heritability of the function and structure of the arterial wall: findings of the Erasmus Rucphen Family (ERF) study," Stroke, vol. 36, no. 11, pp. 2351-2356, 2005.

[11] H. S. Feigelson, M. H. Criqui, A. Fronek, R. D. Langer, and C. A. Molgaard, "Screening for peripheral arterial disease: the sensitivity, specificity, and predictive value of noninvasive tests in a defined population," The American Journal of Epidemiology, vol. 140, no. 6, pp. 526-534, 1994.

[12] A. Yamashina, H. Tomiyama, K. Takeda et al., "Validity, reproducibility, and clinical significance of noninvasive brachial-ankle pulse wave velocity measurement," Hypertension Research, vol. 25, no. 3, pp. 359-364, 2002.

[13] M. Cecelja and P. Chowienczyk, "Role of arterial stiffness in cardiovascular disease," JRSM Cardiovascular Disease, vol. 1, pp. $11-21,2012$.

[14] S. Laurent, P. Boutouyrie, R. Asmar et al., "Aortic stiffness is an independent predictor of all-cause and cardiovascular mortality in hypertensive patients," Hypertension, vol. 37, no. 5, pp. 12361241, 2001.

[15] J. Sugawara, K. Hayashi, T. Yokoi et al., "Brachial-ankle pulse wave velocity: an index of central arterial stiffness?" Journal of Human Hypertension, vol. 19, no. 5, pp. 401-406, 2005.

[16] A. Yamashina, H. Tomiyama, T. Arai et al., "Brachial-ankle pulse wave velocity as a marker of atherosclerotic vascular damage and cardiovascular risk," Hypertension Research, vol. 26, no. 8, pp. 615-622, 2003.

[17] A. P. Avolio, S. G. Chen, R. P. Wang, C. L. Zhang, M. F. Li, and M. F. O'Rourke, "Effects of aging on changing arterial compliance and left ventricular load in a northern Chinese urban community," Circulation, vol. 68, no. 1, pp. 50-58, 1983.

[18] S. S. Franklin, M. J. Jacobs, N. D. Wong, G. J. L'Italien, and P. Lapuerta, "Predominance of isolated systolic hypertension among middle-aged and elderly US hypertensives: analysis based on National Health and Nutrition Examination Survey (NHANES) III," Hypertension, vol. 37, no. 3, pp. 869-874, 2001.
[19] A. Benetos, C. Adamopoulos, J.-M. Bureau et al., "Determinants of accelerated progression of arterial stiffness in normotensive subjects and in treated hypertensive subjects over a 6-year period," Circulation, vol. 105, no. 10, pp. 1202-1207, 2002.

[20] C. Wanner, V. Krane, W. März et al., "Atorvastatin in patients with type 2 diabetes mellitus undergoing hemodialysis," The New England Journal of Medicine, vol. 353, no. 3, pp. 238-248, 2005.

[21] B. I. Freedman, L. Andries, Z. K. Shihabi et al., "Glycated albumin and risk of death and hospitalizations in diabetic dialysis patients," Clinical Journal of the American Society of Nephrology, vol. 6, no. 7, pp. 1635-1643, 2011.

[22] G. A. Block, T. E. Hulbert-Shearon, N. W. Levin, and F. K. Port, "Association of serum phosphorus and calcium x phosphate product with mortality risk in chronic hemodialysis patients: a national study," The American Journal of Kidney Diseases, vol. 31, no. 4, pp. 607-617, 1998. 


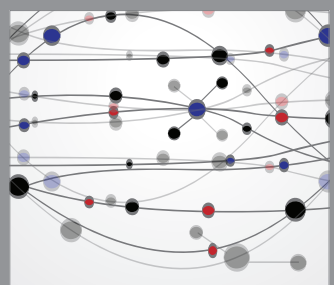

The Scientific World Journal
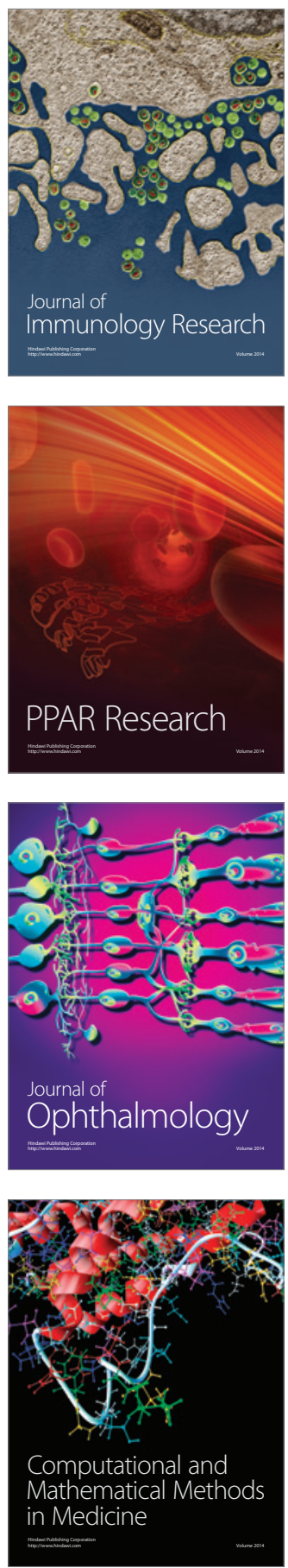

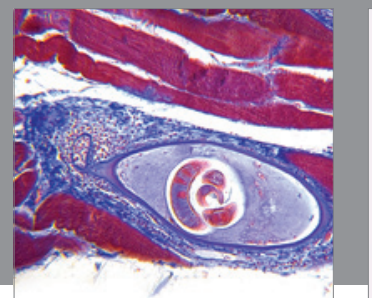

Gastroenterology

Research and Practice
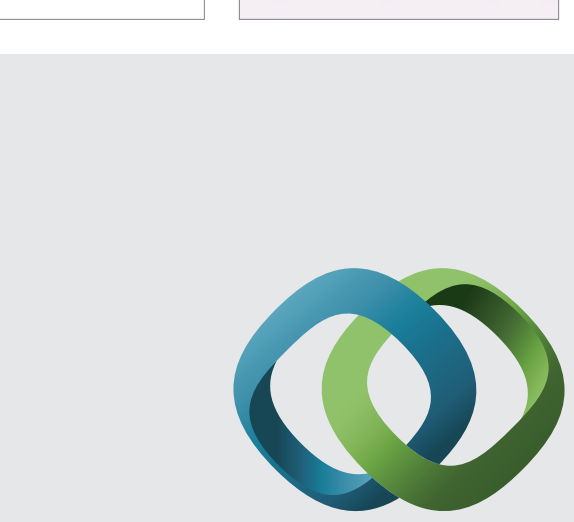

\section{Hindawi}

Submit your manuscripts at

http://www.hindawi.com
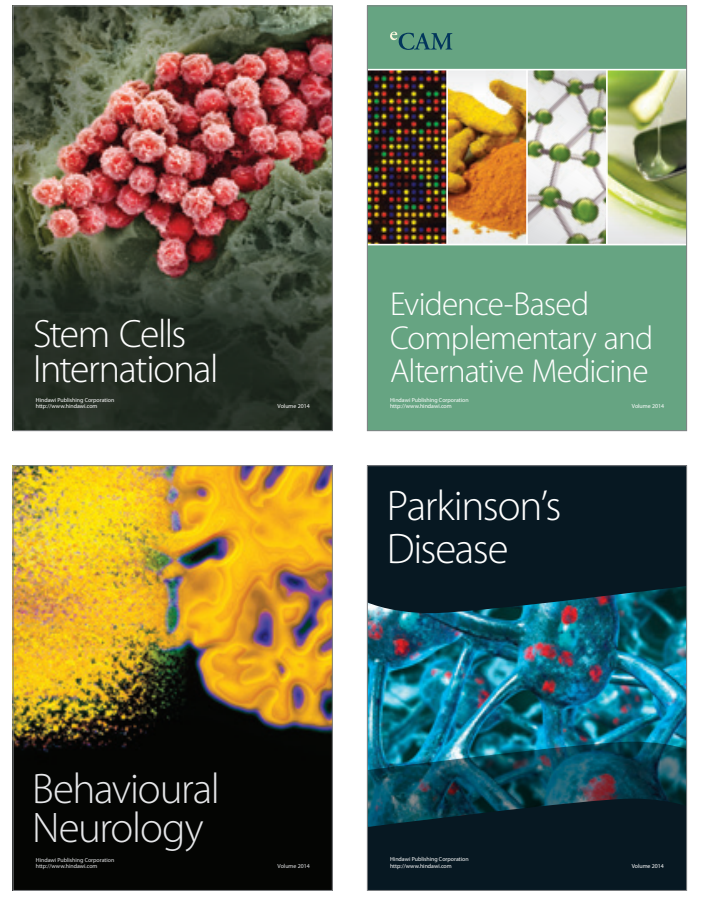
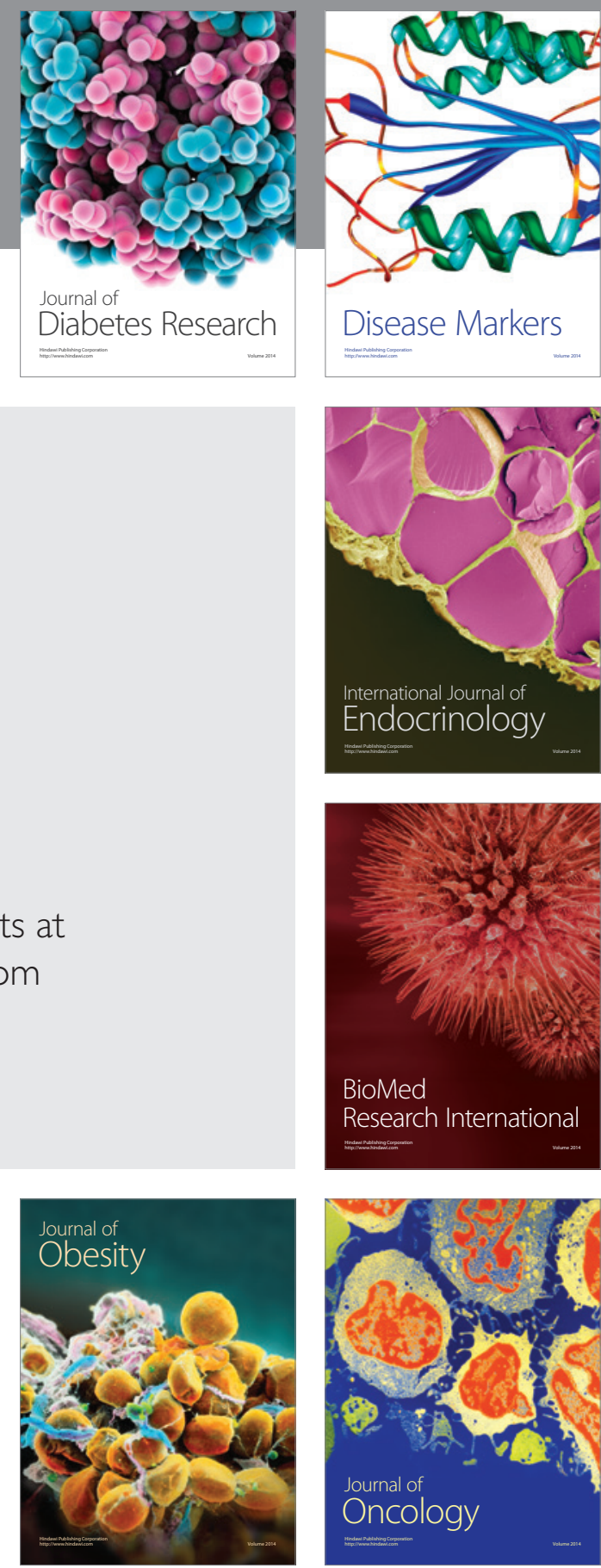

Disease Markers
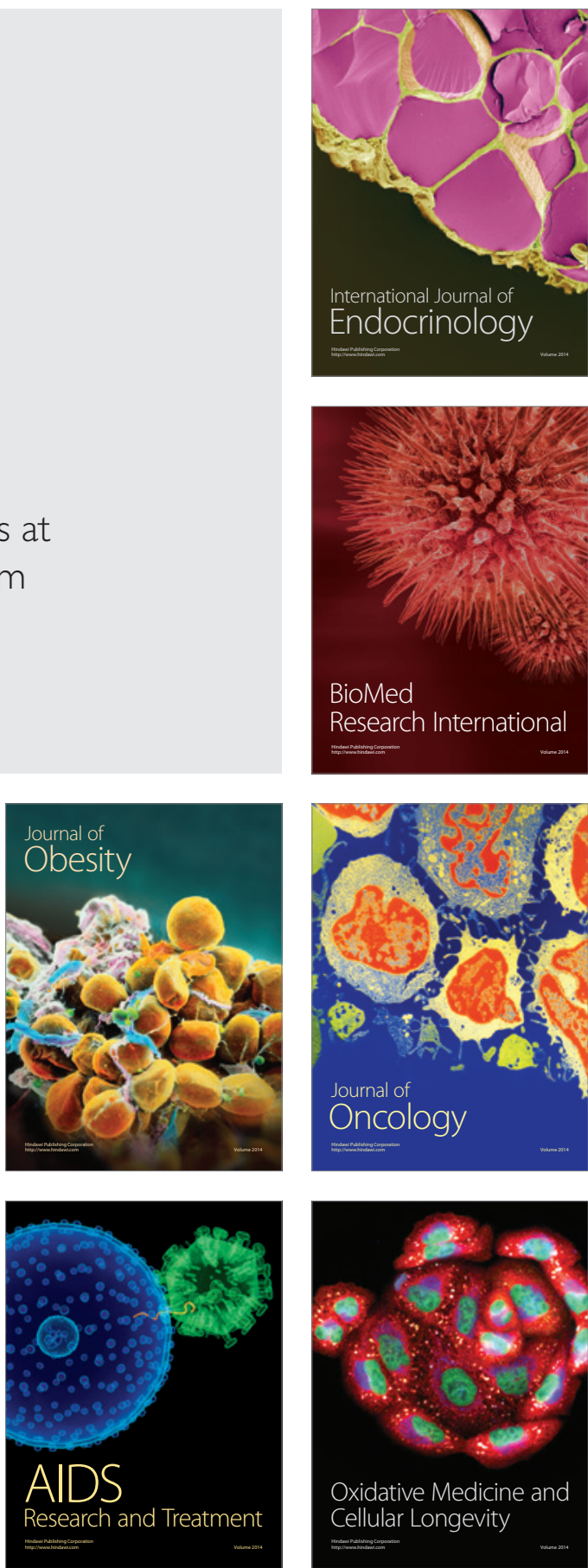